1 Hacettepe Journal of Mathematics and Statistics

holume 47 (1) (2018), $19-35$

\title{
On topological indices of honeycomb networks and graphene networks
}

\author{
Shehnaz Akhter*, Muhammad Imran ${ }^{\dagger}$, Wei $\mathrm{Gao}^{\ddagger}$ and Mohammad Reza Farahani ${ }^{\S}$
}

\begin{abstract}
In QSAR/QSPR study, topological indices such as the Shultz index, generalized Randic index,Zagreb index, general sum-connectivity index, atom-bond connectivity $(A B C)$ index and geometric-arithmetic $(G A)$ index exploited to estimate the bioactivity of chemical compounds. In this paper, we compute first general Zagreb index, general Randić connectivity index, general sum-connectivity index, $A B C, G A, A B C_{4}$ and $G A_{5}$ indices of the line graphs of subdivision graphs of honeycomb networks and graphene.
\end{abstract}

Keywords: Topological indices, atom-bond connectivity index, geometric arithmetic index, honeycomb network, graphene.

2000 AMS Classification: 05C90

Received: 14.11.2016 Accepted : 27.03.2017 Doi : 10.15672/HJMS.2017.464

${ }^{*}$ Department of Mathematics,

School of Natural Sciences (SNS), National University of Sciences and Technology (NUST),

Sector H-12, Islamabad, Pakistan

Email : shehnazakhter36@yahoo.com

${ }^{\dagger}$ Department of Mathematical Sciences, United Arab Emirates University, P. O. Box 15551, $\mathrm{Al}$ Ain, United Arab Emirates

Email : imrandhab@gmail.com

${ }^{\ddagger}$ School of Information Science and Technology, Yunnan Normal University, Kunming 650500, China

Email : gaowei@ynnu.edu.cn

${ }^{\S}$ Department of Applied Mathematics, Iran University of Science and Technology (IUST)

Narmak, 16844, Tehran, Iran

Email : mrfarahani8@gmail.com 


\section{Introduction and preliminary results}

The application of molecular structure indices is nowadays a standard procedure in the study of structure-property relations, especially in QSPR/QSAR study. In the last few years, the number of proposed molecular descriptors is rapidly growing due to the chemical significance of these indices. Randić index has been closely correlated with many chemical properties and found parallel to the boiling point and Kovats constants. The atom-bond connectivity $(A B C)$ index provides a good model for the stability of linear and branched alkanes as well as the strain energy of cycloalkanes. For certain physico-chemical properties, the predictive power of geometric-arithmetic $(G A)$ index is somewhat better than predictive power of the Randić connectivity index. Graph theory has provided chemist with a variety of useful tools, such as topological matrices, topological polynomials and topological indices. A molecular graph is a representation of the structural formula of a chemical compound in terms of graph theory, whose vertices correspond to the atoms of the compound and edges correspond to chemical bonds.

Topological indices are the molecular descriptors that describe the structures of chemical compounds and they help us to predict certain physico-chemical properties like boiling point, enthalpy of vaporization, stability, and so on. A topological index denoted by $\operatorname{Top}(G)$ of a graph $G$ is equal to the topological index $\operatorname{Top}(H)$ of the graph $H$, if and only if two graphs $G$ and $H$ are isomorphic. The Wiener index in the theoretical point of view and applications, is the first and most studied topological index in chemical graph theory. Firstly, the Wiener index has known as path index but later on it was renamed as Wiener index.

Throughout in this paper, $G$ be a connected, simple and undirected graph. In a graph $G$, two vertices are adjacent if and only if they are the end vertices of an edge and two edges are incident to each other if and only if they share a common vertex. The degree of a vertex $u$ is denoted by $d_{u}$ and $s_{u}=\sum_{v \in N_{u}} d_{v}$, where $N_{u}=\{u \in V(G) \mid u v \in E(G)\}$ that is known as the set of neighbor vertices of $u$. The subdivision graph $S(G)$ is the graph attained from $G$ by inserting a vertex on each edge of graph $G$. The line graph $L(G)$ of a graph $G$ is the graph whose vertices are the edges of existing graph $G$. The Randić connectivity index [22] was defined as:

$$
R(G)=\sum_{u v \in E(G)} \frac{1}{\sqrt{d_{u} d_{v}}} .
$$

The generalization of Randić index is known as general Randic connectivity index or general product-connectivity index and defined as follows:

$$
R_{\alpha}(G)=\sum_{u v \in E(G)}\left(d_{u} d_{v}\right)^{\alpha}
$$

where $\alpha$ is a real number. The sum-connectivity index of a graph $G$ was proposed by Zhou [29]. The concept of sum-connectivity index was extended to the general sumconnectivity index [30] and is defined as follows:

$$
\chi_{\alpha}(G)=\sum_{u v \in E(G)}\left(d_{u}+d_{v}\right)^{\alpha},
$$

where $\alpha$ is a real number. Li and Zhao [17] introduced the first general Zagreb index as follows:

$$
M_{\alpha}(G)=\sum_{u \in V(G)}\left(d_{u}\right)^{\alpha},
$$


where $\alpha$ is a real number. The atom-bond Connectivity $A B C$ index was introduced by Estrada et al. [9] and is defined as:

$$
A B C(G)=\sum_{u v \in E(G)} \sqrt{\frac{d_{u}+d_{v}-2}{d_{u} d_{v}}} .
$$

Vukičević [28] introduced the geometric-arithmetic $(G A)$ index and defined as:

$$
G A(G)=\sum_{u v \in E(G)} \frac{2 \sqrt{d_{u} d_{v}}}{d_{u}+d_{v}} .
$$

The fourth member of the class of $A B C$ index was introduced by Ghorbani and Hosseinzadeh [10] in 2010 and defined as:

$$
A B C_{4}(G)=\sum_{u v \in E(G)} \sqrt{\frac{s_{u}+s_{v}-2}{s_{u} s_{v}}} .
$$

Recently fifth version of $G A$ index $G A_{5}$ is proposed by Graovac [11] in 2011 and defined as:

$$
G A_{5}(G)=\sum_{u v \in E(G)} \frac{2 \sqrt{s_{u} s_{v}}}{s_{u}+s_{v}} .
$$

For further study of these topological indices, consult $[1,2,3,4,5,6,7,8,12,13,14$, $15,16,20,21]$.

\section{Topological indices of $L(S(G))$}

Ranjini et al. [25] was calculated the explicit expressions for the Shultz indices of the subdivision graphs of the tadpole, wheel, helm and ladder graphs. They also computed the Zagreb indices of the line graphs of the tadpole, wheel and ladder graphs with subdivision in [24]. Su and Xu [26] calculated the general sum-connectivity indices and co-indices of the line graphs of the tadpole, wheel and ladder graphs with subdivision. Nadeem et al. [18] computed $A B C_{4}$ and $G A_{5}$ indices of the line graph of the tadpole, wheel and ladder graphs by using the notion of subdivision. They extended this study to the topological properties of the line graph of subdivision of certain nanostructures in 2016 [19]. In this paper, we study the topological properties of the line graph of honeycomb networks and Graphene by using the technique of subdivision.

2.1. Honeycomb networks. Honeycomb networks are used in computer graphics, cellular phone base stations, image processing and as a representation of benzenoid hydrocarbons in chemistry. If we recursively use hexagonal tiling in a particular pattern, honeycomb networks are formed. An $n$-dimensional honeycomb network is denoted as $H C_{n}$, where $n$ is the number of hexagons between central and boundary hexagon. Honeycomb network $H C_{n}$ is constructed from $H C_{n-1}$ by adding a layer of hexagons around boundary of $H C_{n-1}$. The number of vertices in honeycomb network $H C_{n}$ are $6 n^{2}$ and number of edges are $9 n^{2}-3 n$. There are four types of edges in $H C_{n}$ based on degree sum of vertices lying at unit distance from end vertices of each edge. A few networks such as hexagonal, honeycomb, and grid networks, for instance, bear resemblance to atomic or molecular lattice structures. These networks have very interesting topological properties which have been studied in different aspects in [15, 23].

The line graph of subdivision graph of honeycomb networks is shown in Figure 2. The number of vertices in the line graph of the subdivision graph of honeycomb networks $H C_{n}$ are $6 n(3 n-1)$ and number of edges are $27 n^{2}-15 n$. In this section, we computed first general Zagreb index, general Randić connectivity index, general sum connectivity index, 


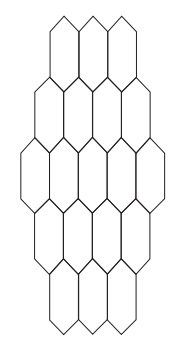

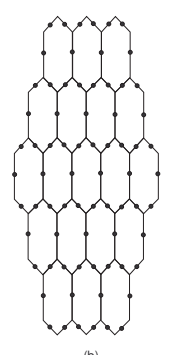

Figure 1. (a) A 2-dimensional honeycomb network. (b) A subdivision 2-dimensional honeycomb network.

$A B C$ index, $G A$ index, $A B C_{4}$ index and $G A_{5}$ index of the line graph of the subdivision graph of honeycomb networks.

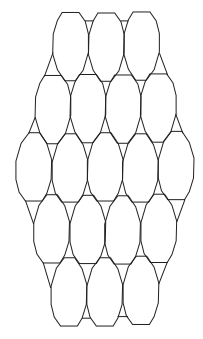

Figure 2. A line graph of subdivision graph of honeycomb network.

\begin{tabular}{|c|c|c|c|}
\hline$\left(d_{u}, d_{v}\right)$ where $u v \in E(G)$ & $(2,2)$ & $(2,3)$ & $(3,3)$ \\
\hline Number of edges & $6(n+1)$ & $12(n-1)$ & $3\left(9 n^{2}-11 n+2\right)$ \\
\hline
\end{tabular}

Table 1. The edge partition of graph $G$ based on degree of end vertices of each edge.

2.1. Theorem. Let $G$ be the line graph of the subdivision graph of honeycomb networks $H C_{n}, n \geq 1$. Then

(1) $M_{\alpha}(G)=18 n(n-1) \cdot 3^{\alpha}+12 n \cdot 2^{\alpha}$,

(2) $R_{\alpha}(G)=12(n-1) \cdot 6^{\alpha}+6(n+1) \cdot 2^{2 \alpha}+3\left(9 n^{2}-11 n+2\right) \cdot 3^{2 \alpha}$,

(3) $\chi_{\alpha}(G)=12(n-1) \cdot 5^{\alpha}+6(n+1) \cdot 2^{2 \alpha}+3\left(9 n^{2}-11 n+2\right) \cdot 6^{\alpha}$,

where $\alpha$ is a real number.

Proof. The graph $G$ have total $6 n(3 n-1)$ vertices among which $18 n(n-1)$ and $12 n$ number of vertices are of degree 3 and 2, respectively. Use these values in the formula of first general Zagreb index and get the required result.

The total number of edges of the line graph of the subdivision graph of honeycomb networks $H C_{n}$ is $3 n(9 n-5)$. Therefore we get the edge partition based on the degree of the vertices as shown in Table 1 . Use Table 1 in the formulae of general Randić index and general sum-connectivity index and compute required results. 


\begin{tabular}{|c|c|}
\hline$\left(s_{u}, s_{v}\right)$ where $u v \in E(G)$ & Number of edges \\
\hline$(4,4)$ & 6 \\
\hline$(4,5)$ & 12 \\
\hline$(5,5)$ & $6(n-2)$ \\
\hline$(5,8)$ & $12(n-1)$ \\
\hline$(8,8)$ & $6(n-1)$ \\
\hline$(8,9)$ & $12(n-1)$ \\
\hline$(9,9)$ & $3\left(9 n^{2}-17 n+8\right)$ \\
\hline
\end{tabular}

Table 2. The edge partition of graph $G$ based on degree sum of neighbor vertices of end vertices of each edge.

2.2. Theorem. Let $G$ be the line graph of the subdivision graph of honeycomb networks $H C_{n}, n \geq 1$. Then its $A B C$ index is equal to

$$
A B C(G)=18 n^{2}+(9 \sqrt{2}-22) n+4-3 \sqrt{2} .
$$

Proof. By the definition of $A B C$ index and using the values from Table 1, we get

$$
\begin{aligned}
A B C(G)= & \sum_{u v \in E(G)} \sqrt{\frac{d_{u}+d_{v}-2}{d_{u} d_{v}}} \\
= & 6(n+1) \sqrt{\frac{2+2-2}{2 \times 2}}+12(n-1) \sqrt{\frac{2+3-2}{2 \times 3}} \\
& +3\left(9 n^{2}-11 n+2\right) \sqrt{\frac{3+3-2}{3 \times 3}} \\
= & 18 n^{2}+(9 \sqrt{2}-22) n+4-3 \sqrt{2} .
\end{aligned}
$$

2.3. Theorem. Let $G$ be the line graph of the subdivision graph of honeycomb networks $H C_{n}, n \geq 1$. Then its $A B C$ index is equal to

$$
G A(G)=27 n^{2}+\left(\frac{24 \sqrt{6}}{5}-27\right) n-\frac{24 \sqrt{6}}{5}+12 .
$$

Proof. From the definition of $G A$ index and by using Table 1, we obtain

$$
\begin{aligned}
G A(G) & =\sum_{u v \in E(G)} \frac{2 \sqrt{d_{u} d_{v}}}{d_{u}+d_{v}} \\
& =6(n+1) \frac{2 \sqrt{2 \times 2}}{2+2}+12(n-1) \frac{2 \sqrt{2 \times 3}}{2+3}+3\left(9 n^{2}-11 n+2\right) \frac{2 \sqrt{3 \times 3}}{3+3} \\
& =27 n^{2}+\left(\frac{24 \sqrt{6}}{5}-27\right) n-\frac{24 \sqrt{6}}{5}+12 .
\end{aligned}
$$

There are seven types of edges on degree based sum of neighbors vertices of each edge in the line graph of the subdivision graph of honeycomb networks $H C_{n}$. We use this partition of edges to calculate $A B C_{4}$ and $G A_{5}$ indices in the following theorems. 
2.4. Theorem. Let $G$ be the line graph of the subdivision graph of honeycomb networks $H C_{n}, n \geq 1$. Then the $A B C_{4}$ index is

$$
A B C_{4}(G)= \begin{cases}3 \sqrt{6}, & n=1 ; \\ 12 n^{2}+\left(\frac{6 \sqrt{8}}{5}+\frac{3 \sqrt{14}}{4}+\frac{6 \sqrt{11}}{\sqrt{10}}+\frac{6 \sqrt{15}}{\sqrt{2}}-\frac{68}{3}\right) n & \\ +\frac{3 \sqrt{6}}{2}-\frac{6 \sqrt{8}}{5}-\frac{3 \sqrt{14}}{4}+\frac{6 \sqrt{7}}{\sqrt{5}}-\frac{6 \sqrt{11}}{\sqrt{10}}-\frac{6 \sqrt{15}}{\sqrt{2}}+\frac{32}{3}, & n \neq 1 .\end{cases}
$$

Proof. The edge types of the line graph of the subdivision graph of honeycomb networks $H C_{n}$ based on degree sum of vertices lying at unit distance from end vertices of each edge shown in Table 2. Now we can apply formula of $A B C_{4}$ index.

Case 1. For $n \neq 1$,

$$
\begin{aligned}
A B C_{4}(G)= & \sum_{u v \in E(G)} \sqrt{\frac{s_{u}+s_{v}-2}{s_{u} s_{v}}} \\
= & 6 \sqrt{\frac{4+4-2}{4 \times 4}}+12 \sqrt{\frac{5+4-2}{4 \times 5}}+6(n-2) \sqrt{\frac{5+5-2}{5 \times 5}} \\
+ & 12(n-1) \sqrt{\frac{5+8-2}{5 \times 8}}+6(n-1) \sqrt{\frac{8+8-2}{8 \times 8}} \\
& +12(n-1) \sqrt{\frac{8+9-2}{8 \times 9}}+3\left(9 n^{2}-17 n+8\right) \sqrt{\frac{9+9-2}{9 \times 9}} \\
= & 12 n^{2}+\left(\frac{6 \sqrt{8}}{5}+\frac{3 \sqrt{14}}{4}+\frac{6 \sqrt{11}}{\sqrt{10}}+\frac{6 \sqrt{15}}{\sqrt{2}}-\frac{68}{3}\right) n+\frac{3 \sqrt{6}}{2}-\frac{6 \sqrt{8}}{5} \\
& -\frac{3 \sqrt{14}}{4}+\frac{6 \sqrt{7}}{\sqrt{5}}-\frac{6 \sqrt{11}}{\sqrt{10}}-\frac{6 \sqrt{15}}{\sqrt{2}}+\frac{32}{3} .
\end{aligned}
$$

Case 2. For $n=1$,

$$
\begin{aligned}
A B C_{4}(G) & =\sum_{u v \in E(G)} \sqrt{\frac{s_{u}+s_{v}-2}{s_{u} s_{v}}} \\
& =12 \times \sqrt{\frac{4+4-2}{4 \times 4}} \\
& =3 \sqrt{6} .
\end{aligned}
$$

2.5. Theorem. Let $G$ be the line graph of the subdivision graph of honeycomb networks $H C_{n}, n \geq 1$. Then the $G A_{5}$ index is

$$
G A_{5}(G)= \begin{cases}6, & n=1 \\ 27 n^{2}+\left(\frac{48 \sqrt{10}}{13}-39\right) n+\frac{144 \sqrt{2}}{17}-\frac{4 \sqrt{10}}{13}+\frac{16 \sqrt{5}}{3}+12, & n \neq 1\end{cases}
$$

Proof. The edge types of the line graph of the subdivision graph of honeycomb networks $H C_{n}$ based on degree sum of vertices lying at unit distance from end vertices of each edge shown in Table 2. Now we can apply formula of $G A_{5}$ index.

Case 1. For $n \neq 1$, Since

$$
G A_{5}(G)=\sum_{u v \in E(G)} \frac{2 \sqrt{s_{u} s_{v}}}{s_{u}+s_{v}}
$$




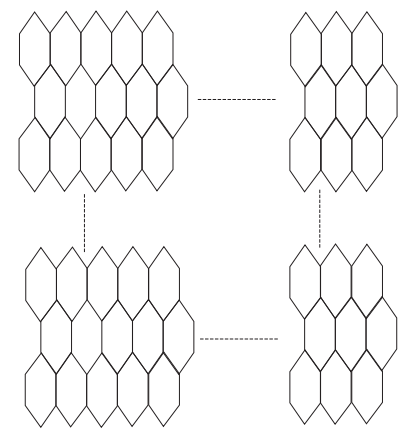

Figure 3. Graphene $G_{t, s}$

By using Table 2,

$$
\begin{aligned}
G A_{5}(G)= & 6\left(\frac{2 \sqrt{4 \times 4}}{4+4}\right)+12\left(\frac{2 \sqrt{4 \times 5}}{4+5}\right)+6(n-2)\left(\frac{2 \sqrt{5 \times 5}}{5+5}\right) \\
& +12(n-1)\left(\frac{2 \sqrt{5 \times 8}}{5+8}\right)+6(n-1)\left(\frac{2 \sqrt{8 \times 8}}{8+8}\right) \\
& +12(n-1)\left(\frac{2 \sqrt{8 \times 9}}{8+9}\right)+3\left(9 n^{2}-17 n+8\right)\left(\frac{2 \sqrt{9 \times 9}}{9+9}\right) \\
= & 27 n^{2}+\left(\frac{48 \sqrt{10}}{13}-39\right) n+\frac{144 \sqrt{2}}{17}-\frac{4 \sqrt{10}}{13}+\frac{16 \sqrt{5}}{3}+12 .
\end{aligned}
$$

Case 2. For $n=1$,

$$
\begin{aligned}
G A_{5}(G) & =\sum_{u v \in E(G)} \frac{2 \sqrt{s_{u} s_{v}}}{s_{u}+s_{v}} \\
& =12 \times \frac{2 \sqrt{4 \times 4}}{4+4} \\
& =6 .
\end{aligned}
$$

2.2. Graphene. Graphene is an atomic scale honeycomb lattice made of the carbon atoms. It is the first $2 D$ material which was isolated from graphite in 2004 by Professor Andre Geim and Professor Kostya Novoselov. Graphene is 200 times stronger than steel, one million times thinner than a human hair, and world's most conductive material. So it has captured the attention of scientists, researchers, and industries worldwide. It is one of the most promising nanomaterials because of its unique combination of super properties, which opens a way for its exploitation in a wide spectrum of applications ranging from electronics to optics, sensors, and biodevices. Also it is the most effective material for electromagnetic interference (EMI) shielding. Sridhara was calculated some topological indices of graphene in [27].

The subdivision graph of graphene $G_{t, s}$ and its line graph are shown in Figures 4. The number of vertices in the line graph of the subdivision graph of Graphene $G_{t, s}$ are $2(3 s t+2 s+2 t-1)$ and number of edges are $(9 s t+4 s+4 t-5)$. In this section, we computed first general Zagreb index, general Randić connectivity index, general sum connectivity index, $A B C$ index, $G A$ index, $A B C_{4}$ index and $G A_{5}$ index of the line graph of the subdivision graph of graphene $G_{t, s}$. 


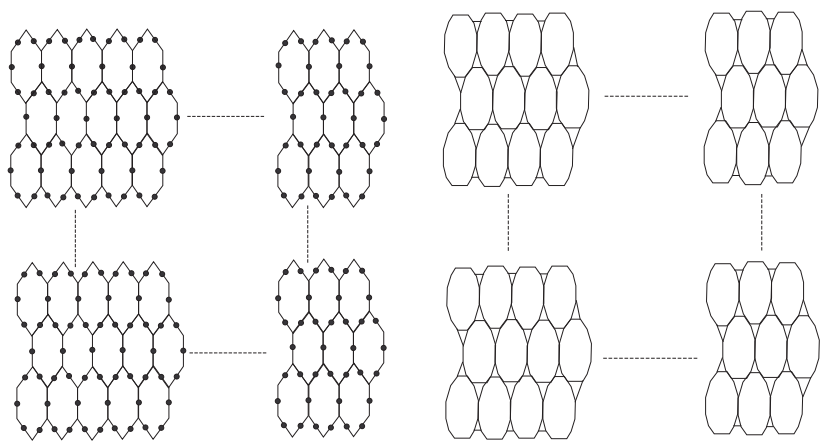

Figure 4. (a) A subdivision graph of graphene $G_{t, s}$, (b) A line graph of subdivision graph of graphene $G_{t, s}$

\begin{tabular}{|c|c|c|}
\hline Row & $n_{2}$ & $n_{3}$ \\
\hline 1 & $2(s+3)$ & $4(2 s-1)$ \\
\hline 2 & 4 & $6 s$ \\
\hline 3 & 4 & $6 s$ \\
\hline 4 & 4 & $6 s$ \\
\hline$\vdots$ & $\vdots$ & $\vdots$ \\
\hline$t$ & $2(s+3)$ & $2(2 s-1)$ \\
\hline Total & $4(s+t+1)$ & $6(s t-1)$ \\
\hline
\end{tabular}

Table 3. The vertex partition of graph $G$ based on degree of vertices.

2.6. Theorem. The first general Zagreb index of the line graph of the subdivision graph of graphene with $t$ rows of benzene rings and $s$ benzene rings in each row is given by

$$
M_{\alpha}(G)= \begin{cases}(s+2) 2^{\alpha+2}+2(s-1) 3^{\alpha+1}, & t=1 \\ (s+t+1) 2^{\alpha+2}+2(s t-1) 3^{\alpha+1}, & t \neq 1\end{cases}
$$

Proof. Case 1. For $t \neq 1$,

The line graph of the subdivision graph of graphene have total $2(3 s t+2 s+2 t-1)$ vertices among which $4(s+t+1)$ and $6(s t-1)$ number of vertices are of degree 2 and 3 , respectively. Use these values in the formula of first general Zagreb index and obtain the required result for $t \neq 1$.

Case 2. For $t=1$,

The line graph of the subdivision graph of graphene have total $2(5 s+1)$ vertices among which $4(s+2)$ and $6(s-1)$ number of vertices are of degree 2 and 3 , respectively. Use these values in the formula of first general Zagreb index and obtain the required result for $t=1$.

2.7. Theorem. Let $G$ be the line graph of the subdivision graph of graphene with $t$ rows of benzene rings and $s$ benzene rings in each row. Then

$$
R_{\alpha}(G)= \begin{cases}(s+5) 2^{2 \alpha+1}+4(s-1) 6^{\alpha}+7(s-1) 3^{2 \alpha}, & t=1 \\ (2 s+3 t+6) 2^{2 \alpha}+2(2 s+t-2) 6^{\alpha}+(9 s t-2 s-t-7) 3^{2 \alpha}, & t \neq 1\end{cases}
$$




\begin{tabular}{|c|c|c|c|}
\hline Row & $m_{2,2}$ & $m_{2,3}$ & $m_{3,3}$ \\
\hline 1 & $s+6$ & $2 s$ & $9 s-5$ \\
\hline 2 & 3 & 2 & $9 s-1$ \\
\hline 3 & 3 & 2 & $9 s-1$ \\
\hline 4 & 3 & 2 & $9 s-1$ \\
\hline$\vdots$ & $\vdots$ & $\vdots$ & $\vdots$ \\
\hline$t$ & $s+6$ & $2 s$ & $7 s-4$ \\
\hline Total & $2 s+3 t+6$ & $2(2 s+t-2)$ & $9 s t-2 s-t-7$ \\
\hline
\end{tabular}

Table 4. The edge partition of graph $G$ based on degree of end vertices of each edge.

\begin{tabular}{|c|c|c|c|}
\hline$\left(d_{u}, d_{v}\right)$ where $u v \in E(G)$ & $m_{2,2}$ & $m_{2,3}$ & $m_{3,3}$ \\
\hline Number of edges & $2(s+5)$ & $4(s-1)$ & $7(s-1)$ \\
\hline
\end{tabular}

Table 5. The edge partition of graph $G$ based on degree of end vertices of each edge for $t=1$.

where $\alpha$ is a real number.

Proof. From the formula of general Randić index and Table 4 and 5.

Case 1. For $t \neq 1$,

$$
\begin{aligned}
R_{\alpha}(G) & =\sum_{u v \in E(G)}\left(d_{u} d_{v}\right)^{\alpha} \\
& =m_{2,2}(2 \times 2)^{\alpha}+m_{2,3}(2 \times 3)^{\alpha}+m_{3,3}(3 \times 3)^{\alpha}
\end{aligned}
$$

By using Table 4, we get

$$
R_{\alpha}(G)=(2 s+3 t+6) 2^{2 \alpha}+2(2 s+t-2) 6^{\alpha}+(9 s t-2 s-t-7) 3^{2 \alpha} .
$$

Case 2. For $t=1$,

$$
\begin{aligned}
R_{\alpha}(G) & =\sum_{u v \in E(G)}\left(d_{u} d_{v}\right)^{\alpha} \\
& =m_{2,2}(2 \times 2)^{\alpha}+m_{2,3}(2 \times 3)^{\alpha}+m_{3,3}(3 \times 3)^{\alpha}
\end{aligned}
$$

By using Table 5, we get

$$
\begin{aligned}
R_{\alpha}(G) & =2(s+5) 4^{\alpha}+4(s-1) 6^{\alpha}+7(s-1) 9^{\alpha} \\
& =(s+5) 2^{2 \alpha+1}+4(s-1) 6^{\alpha}+7(s-1) 3^{2 \alpha} .
\end{aligned}
$$

2.8. Theorem. Let $G$ be the line graph of the subdivision graph of graphene with $t$ rows of benzene rings and $s$ benzene rings in each row. Then

$$
\chi_{\alpha}(G)= \begin{cases}(s+5) 2^{2 \alpha+1}+4(s-1) 5^{\alpha}+7(s-1) 6^{\alpha}, & t=1 \\ (2 s+3 t+6) 2^{2 \alpha}+2(2 s+t-2) 5^{\alpha}+(9 s t-2 s-t-7) 6^{\alpha}, & t \neq 1\end{cases}
$$

where $\alpha$ is a real number. 
Proof. The edge types of the line graph of the subdivision graph of graphene based on the degree of end vertices of each edge shown in the Table 4 and 5. Now we can apply formula of general sum-connectivity index.

Case 1. For $t \neq 1$,

$$
\begin{aligned}
\chi_{\alpha}(G) & =\sum_{u v \in E(G)}\left(d_{u}+d_{v}\right)^{\alpha} \\
& =m_{2,2}(2+2)^{\alpha}+m_{2,3}(2+3)^{\alpha}+m_{3,3}(3+3)^{\alpha}
\end{aligned}
$$

By using Table 4, we get

$$
\chi_{\alpha}(G)=(2 s+3 t+6) 2^{2 \alpha}+2(2 s+t-2) 5^{\alpha}+(9 s t-2 s-t-7) 6^{\alpha} .
$$

Case 2. For $t=1$,

$$
\begin{aligned}
\chi_{\alpha}(G) & =\sum_{u v \in E(G)}\left(d_{u}+d_{v}\right)^{\alpha} \\
& =m_{2,2}(2+2)^{\alpha}+m_{2,3}(2+3)^{\alpha}+m_{3,3}(3+3)^{\alpha}
\end{aligned}
$$

By using Table 5, we get

$$
\begin{aligned}
\chi_{\alpha}(G) & =2(s+5) 4^{\alpha}+4(s-1) 5^{\alpha}+7(s-1) 6^{\alpha} \\
& =(s+5) 2^{2 \alpha+1}+4(s-1) 5^{\alpha}+7(s-1) 6^{\alpha} .
\end{aligned}
$$

2.9. Theorem. The ABC index of the line graph of the subdivision graph of graphene with $t$ rows of benzene rings and $s$ benzene rings in each row is given by

$$
A B C(G)= \begin{cases}\frac{2(9+7 \sqrt{2}) s+2(9-7 \sqrt{2})}{3 \sqrt{2}}, & t=1 \\ \frac{18 \sqrt{2} s t+(18-4 \sqrt{2}) s+(15-2 \sqrt{2}) t+6-14 \sqrt{2}}{3 \sqrt{2}}, & t \neq 1 .\end{cases}
$$

Proof. Consider a graphene with $t$ rows and $s$ benzene rings in each row. Let $m_{i, j}$ denotes the number of edges connecting the vertices of degrees $d_{i}$ and $d_{j}$. Two-dimensional structure of the line graph of the subdivision graph of graphene contains only $m_{2,2}, m_{2,3}$ and $m_{3,3}$ types of edges.

Case 1. The $A B C$ index of the line graph of the subdivision graph of graphene for $t \neq 1$ and using Table 4 , we obtain

$$
\begin{aligned}
A B C(G) & =\sum_{u v \in E(G)} \sqrt{\frac{d_{u}+d_{v}-2}{d_{u} d_{v}}} \\
& =m_{2,2} \sqrt{\frac{2+2-2}{2 \times 2}}+m_{2,3} \sqrt{\frac{2+3-2}{2 \times 3}}+m_{3,3} \sqrt{\frac{3+3-2}{3 \times 3}} \\
& =(2 s+3 t+6) \frac{1}{\sqrt{2}}+2(2 s+t-2) \frac{1}{\sqrt{2}}+(9 s t-2 s-t-7) \frac{2}{3} \\
& =\frac{18 \sqrt{2} s t+(18-4 \sqrt{2}) s+(15-2 \sqrt{2}) t+6-14 \sqrt{2}}{3 \sqrt{2}} .
\end{aligned}
$$

Case 2. For $t=1$,

$$
\begin{aligned}
A B C(G) & =\sum_{u v \in E(G)} \sqrt{\frac{d_{u}+d_{v}-2}{d_{u} d_{v}}} \\
& =m_{2,2} \sqrt{\frac{2+2-2}{2 \times 2}}+m_{2,3} \sqrt{\frac{2+3-2}{2 \times 3}}+m_{3,3} \sqrt{\frac{3+3-2}{3 \times 3}}
\end{aligned}
$$


By using Table 5, we get

$$
\begin{aligned}
A B C(G) & =72(s+5) \frac{1}{\sqrt{2}}+4(s-1) \frac{1}{\sqrt{2}}+7(s-1) \frac{2}{3} \\
& =\frac{2(9+7 \sqrt{2}) s+2(9-7 \sqrt{2})}{3 \sqrt{2}}
\end{aligned}
$$

2.10. Theorem. The GA index of the line graph of the subdivision graph of graphene with $t$ rows of benzene rings and $s$ benzene rings in each row is given by

$$
G A(G)= \begin{cases}\frac{(45+8 \sqrt{6}) s+(15-8 \sqrt{6})}{5}, & t=1 \\ \frac{45 s t+4 \sqrt{6} s+2(2 \sqrt{6}+5) t-5}{5}, & t \neq 1\end{cases}
$$

Proof. Case 1. The $G A$ index of the line graph of the subdivision graph of graphene for $t \neq 1$

$$
\begin{aligned}
G A(G) & =\sum_{u v \in E(G)} \frac{2 \sqrt{d_{u} d_{v}}}{d_{u}+d_{v}} \\
& =m_{2,2} \frac{2 \sqrt{2 \times 2}}{2+2}+m_{2,3} \frac{2 \sqrt{2 \times 3}}{2+3}+m_{3,3} \frac{2 \sqrt{3 \times 3}}{3+3}
\end{aligned}
$$

By using Table 4, we get

$$
\begin{aligned}
G A(G) & =(2 s+3 t+6) \frac{2 \times 2}{4}+2(2 s+t-2) \frac{2 \sqrt{6}}{5}+(9 s t-2 s-t-7) \frac{2 \times 3}{6} \\
& =\frac{45 s t+4 \sqrt{6} s+(4 \sqrt{6}+10) t-5}{5} .
\end{aligned}
$$

Case 2. For $t=1$,

$$
\begin{aligned}
G A(G) & =\sum_{u v \in E(G)} \frac{2 \sqrt{d_{u} d_{v}}}{d_{u}+d_{v}} \\
& =m_{2,2} \frac{2 \sqrt{2 \times 2}}{2+2}+m_{2,3} \frac{2 \sqrt{2 \times 3}}{2+3}+m_{3,3} \frac{2 \sqrt{3 \times 3}}{3+3}
\end{aligned}
$$

By using Table 5, we get

$$
\begin{aligned}
G A(G) & =2(s+5) \frac{2 \times 2}{4}+4(s-1) \frac{2 \sqrt{6}}{5}+7(s-1) \frac{2 \times 3}{6} \\
& =\frac{(45+8 \sqrt{6}) s+(15-8 \sqrt{6})}{5} .
\end{aligned}
$$

2.11. Theorem. The fourth atom-bond connectivity index of the line graph of the subdivision graph of graphene with $t$ rows of benzene rings and $s$ benzene rings in each row 


\begin{tabular}{|c|c|c|c|c|c|c|c|}
\hline Row & $e_{4,4}$ & $e_{4,5}$ & $e_{5,5}$ & $e_{5,8}$ & $e_{8,8}$ & $e_{8,9}$ & $e_{9,9}$ \\
\hline 1 & 4 & 4 & $s-2$ & $2 s$ & $s$ & $2 s$ & $6 s-5$ \\
\hline 2 & 1 & 2 & 0 & 2 & 0 & 4 & $9 s-5$ \\
\hline 3 & 1 & 2 & 0 & 2 & 0 & 4 & $9 s-5$ \\
\hline 4 & 1 & 2 & 0 & 2 & 0 & 4 & $9 s-5$ \\
\hline$\vdots$ & $\vdots$ & $\vdots$ & $\vdots$ & $\vdots$ & $\vdots$ & $\vdots$ & $\vdots$ \\
\hline$t$ & 4 & 4 & $s-2$ & $2 s$ & $s$ & $2 s$ & $4(s-1)$ \\
\hline Total & $t+6$ & $2(t+2)$ & $2(s-2)$ & $2(2 s+t-2)$ & $2 s$ & $4(s+t-2)$ & $9 s t-8 s-5 t+1$ \\
\hline
\end{tabular}

Table 6. The edge partition of graph $G$ based on degree sum of neighbor vertices of end vertices of each edge for $t \neq 1$ and $s>1$.

\begin{tabular}{|c|c|c|c|c|c|c|}
\hline Row & $e_{4,4}$ & $e_{4,5}$ & $e_{5,8}$ & $e_{8,8}$ & $e_{8,9}$ & $e_{9,9}$ \\
\hline 1 & 5 & 2 & 2 & 1 & 2 & 1 \\
\hline 2 & 1 & 2 & 2 & 0 & 4 & 4 \\
\hline 3 & 1 & 2 & 2 & 0 & 4 & 4 \\
\hline 4 & 1 & 2 & 2 & 0 & 4 & 4 \\
\hline$\vdots$ & $\vdots$ & $\vdots$ & $\vdots$ & $\vdots$ & $\vdots$ & $\vdots$ \\
\hline$t$ & 5 & 2 & 2 & 1 & 2 & 0 \\
\hline Total & $t+8$ & $2 t$ & $2 t$ & 2 & $4(t-1)$ & $4 t-7$ \\
\hline
\end{tabular}

Table 7. The edge partition of graph $G$ based on degree sum of neighbor vertices of end vertices of each edge for $t \neq 1$ and $s=1$.

\begin{tabular}{|c|c|c|c|c|c|c|c|}
\hline$\left(s_{u}, s_{v}\right)$ where $u v \in E(G)$ & $e_{4,4}$ & $e_{4,5}$ & $e_{5,5}$ & $e_{5,8}$ & $e_{8,8}$ & $e_{8,9}$ & $e_{9,9}$ \\
\hline Number of edges & 10 & 4 & $2(s-2)$ & $4(s-1)$ & $2(s-1)$ & $4(s-1)$ & $s-1$ \\
\hline
\end{tabular}

Table 8. The edge partition of graph $G$ based on degree sum of neighbor vertices of end vertices of each edge for $t=1$ and $s>1$.

is given by

$$
A B C_{4}(G)= \begin{cases}\left(\frac{4 \sqrt{2}}{5}+\frac{\sqrt{14}}{4}+\frac{2 \sqrt{11}}{\sqrt{10}}+\frac{\sqrt{30}}{3}+\frac{4}{9}\right) s+\frac{5 \sqrt{3}}{\sqrt{2}} & \\ -\frac{8 \sqrt{2}}{5}-\frac{\sqrt{14}}{4}-\frac{2 \sqrt{11}}{\sqrt{10}}-\frac{\sqrt{30}}{3}-\frac{4}{9}+\frac{2 \sqrt{7}}{\sqrt{5}}, & t=1, s>1 \\ \left(\frac{\sqrt{3}}{2 \sqrt{2}}+\frac{\sqrt{7}}{\sqrt{5}}+\frac{\sqrt{11}}{\sqrt{10}}+\frac{\sqrt{30}}{3}+\frac{8}{9}\right) t & t \neq 1, s=1 \\ +\frac{4 \sqrt{3}}{\sqrt{2}}-\frac{\sqrt{30}}{3}-\frac{28}{9}, & \\ 4 s t+\left(\frac{4 \sqrt{2}}{5}+\frac{2 \sqrt{11}}{\sqrt{10}}+\frac{\sqrt{14}}{4}+\frac{\sqrt{30}}{3}-\frac{32}{9}\right) s & \\ +\left(\frac{\sqrt{3}}{2 \sqrt{2}}+\frac{\sqrt{7}}{\sqrt{5}}+\frac{\sqrt{11}}{\sqrt{10}}+\frac{\sqrt{30}}{3}-\frac{20}{9}\right) t & \\ +\frac{3 \sqrt{3}}{\sqrt{2}}+\frac{2 \sqrt{7}}{\sqrt{5}}-\frac{8 \sqrt{2}}{5}-\frac{2 \sqrt{11}}{\sqrt{10}}-\frac{2 \sqrt{30}}{3}+\frac{4}{9}, & t \neq 1, s>1 ; \\ 3 \sqrt{6}, & t=1, s=1 .\end{cases}
$$

Proof. Let $e_{i, j}$ denotes the number of edges of the line graph of the subdivision graph of graphene with $i=s_{u}$ and $j=s_{v}$. It is easy to see that the summation of degree of edge endpoints of graphene has seven edge types $e_{4,4}, e_{4,5}, e_{5,5}, e_{5,8}, e_{8,8}, e_{8,9}$ and $e_{9,9}$ that 
are shown in Table 8.

Case 1. The fourth $A B C$ index of the line graph of the subdivision graph of Graphene for $t=1$ and $s>1$,

$$
\begin{aligned}
A B C_{4}(G)= & \sum_{u v \in E(G)} \sqrt{\frac{s_{u}+s_{v}-2}{s_{u} s_{v}}} \\
= & e_{4,4} \sqrt{\frac{4+4-2}{4 \times 4}}+e_{4,5} \sqrt{\frac{4+5-2}{4 \times 5}}+e_{5,5} \sqrt{\frac{5+5-2}{5 \times 5}}+e_{5,8} \sqrt{\frac{5+8-2}{5 \times 8}} \\
& +e_{8,8} \sqrt{\frac{8+8-2}{8 \times 8}}+e_{8,9} \sqrt{\frac{8+9-2}{8 \times 9}}+e_{9,9} \sqrt{\frac{9+9-2}{9 \times 9}}
\end{aligned}
$$

By using Table 8, we get

$$
\begin{aligned}
A B C_{4}(G)= & 10\left(\frac{\sqrt{3}}{2 \sqrt{2}}\right)+4\left(\frac{\sqrt{7}}{2 \sqrt{5}}\right)+2(s-2) \frac{2 \sqrt{2}}{5}+4(s-1) \frac{\sqrt{11}}{2 \sqrt{10}}+2(s-1) \frac{\sqrt{14}}{8} \\
& +4(s-1) \frac{\sqrt{15}}{6 \sqrt{2}}+(s-1) \frac{4}{9} \\
= & \left(\frac{4 \sqrt{2}}{5}+\frac{\sqrt{14}}{4}+\frac{2 \sqrt{11}}{\sqrt{10}}+\frac{\sqrt{30}}{3}+\frac{4}{9}\right) s+\frac{5 \sqrt{3}}{\sqrt{2}}-\frac{8 \sqrt{2}}{5}-\frac{\sqrt{14}}{4}-\frac{2 \sqrt{11}}{\sqrt{10}} \\
& -\frac{\sqrt{30}}{3}-\frac{4}{9}+\frac{2 \sqrt{7}}{\sqrt{5}} .
\end{aligned}
$$

Case 2. For $t \neq 1$ and $s=1$, the line graph of the subdivision graph of graphene has six types of edges, namely $e_{4,4}, e_{4,5}, e_{5,8}, e_{8,8}, e_{8,9}$ and $e_{9,9}$. The number of edges of these types is shown in Table 7. Then

$$
\begin{aligned}
A B C_{4}(G)= & \sum_{u v \in E(G)} \sqrt{\frac{s_{u}+s_{v}-2}{s_{u} s_{v}}} \\
= & e_{4,4} \sqrt{\frac{4+4-2}{4 \times 4}}+e_{4,5} \sqrt{\frac{4+5-2}{4 \times 5}}+e_{5,8} \sqrt{\frac{5+8-2}{5 \times 8}} \\
& +e_{8,8} \sqrt{\frac{8+8-2}{8 \times 8}}+e_{8,9} \sqrt{\frac{8+9-2}{8 \times 9}}+e_{9,9} \sqrt{\frac{9+9-2}{9 \times 9}}
\end{aligned}
$$

By using Table 7 , we get

$$
\begin{aligned}
A B C_{4}(G) & =(t+8) \frac{\sqrt{3}}{2 \sqrt{2}}+2 t \frac{\sqrt{7}}{2 \sqrt{5}}+2 t \frac{\sqrt{11}}{2 \sqrt{10}}+\frac{2 \sqrt{14}}{8}+4(t-1) \frac{\sqrt{15}}{6 \sqrt{2}}+(4 t-7) \frac{4}{9} \\
& =\left(\frac{\sqrt{3}}{2 \sqrt{2}}+\frac{\sqrt{7}}{\sqrt{5}}+\frac{\sqrt{11}}{\sqrt{10}}+\frac{\sqrt{30}}{3}+\frac{8}{9}\right) t+\frac{4 \sqrt{3}}{\sqrt{2}}-\frac{\sqrt{30}}{3}-\frac{28}{9} .
\end{aligned}
$$

Case 3. For $t \neq 1$ and $s>1$, the line graph of the subdivision graph of graphene has seven types of edges, namely $e_{4,4}, e_{4,5}, e_{5,5}, e_{5,8}, e_{8,8}, e_{8,9}$ and $e_{9,9}$. The number of edges of these types is shown in Table 6 . Then

$$
\begin{aligned}
A B C_{4}(G)= & \sum_{u v \in E(G)} \sqrt{\frac{s_{u}+s_{v}-2}{s_{u} s_{v}}} \\
= & e_{4,4} \sqrt{\frac{4+4-2}{4 \times 4}}+e_{4,5} \sqrt{\frac{4+5-2}{4 \times 5}}+e_{5,5} \sqrt{\frac{5+5-2}{5 \times 5}}+e_{5,8} \sqrt{\frac{5+8-2}{5 \times 8}} \\
& +e_{8,8} \sqrt{\frac{8+8-2}{8 \times 8}}+e_{8,9} \sqrt{\frac{8+9-2}{8 \times 9}}+e_{9,9} \sqrt{\frac{9+9-2}{9 \times 9}}
\end{aligned}
$$


By using Table 6, we get

$$
\begin{aligned}
A B C_{4}(G)= & (t+6) \frac{\sqrt{3}}{2 \sqrt{2}}+2(t+2) \frac{\sqrt{7}}{2 \sqrt{5}}+2(s-2) \frac{2 \sqrt{2}}{5}+2(2 s+t-2) \frac{\sqrt{11}}{2 \sqrt{10}} \\
& +2 s \frac{\sqrt{14}}{8}+4(s+t-2) \frac{\sqrt{15}}{6 \sqrt{2}}+(9 s t-8 s-5 t+1) \frac{4}{9} \\
= & 4 s t+\left(\frac{4 \sqrt{2}}{5}+\frac{2 \sqrt{11}}{\sqrt{10}}+\frac{\sqrt{14}}{4}+\frac{\sqrt{30}}{3}-\frac{32}{9}\right) s+\left(\frac{\sqrt{3}}{2 \sqrt{2}}+\frac{\sqrt{7}}{\sqrt{5}}+\frac{\sqrt{11}}{\sqrt{10}}\right. \\
& \left.+\frac{\sqrt{30}}{3}-\frac{20}{9}\right) t+\frac{3 \sqrt{3}}{\sqrt{2}}+\frac{2 \sqrt{7}}{\sqrt{5}}-\frac{8 \sqrt{2}}{5}-\frac{2 \sqrt{11}}{\sqrt{10}}-\frac{2 \sqrt{30}}{3}+\frac{4}{9} .
\end{aligned}
$$

Case 4. The fourth $A B C$ index of the line graph of the subdivision graph of graphene for $t=1$ and $s=1$,

$$
\begin{aligned}
A B C_{4}(G) & =\sum_{u v \in E(G)} \sqrt{\frac{s_{u}+s_{v}-2}{s_{u} s_{v}}} \\
& =e_{4,4} \sqrt{\frac{4+4-2}{4 \times 4}} \\
& =12\left(\frac{\sqrt{3}}{2 \sqrt{2}}\right) \\
& =3 \sqrt{6} .
\end{aligned}
$$

2.12. Theorem. The fifth $G A$ index of the line graph of the subdivision graph of graphene with $t$ rows of benzene rings and $s$ benzene rings in each row is given by

$$
G A_{5}(G)= \begin{cases}12, & t=1, s=1 \\ \left(5+\frac{16 \sqrt{10}}{13}+\frac{48 \sqrt{2}}{17}\right) s+3+\frac{16 \sqrt{5}}{9} & t=1, s>1 \\ -\frac{16 \sqrt{10}}{13}-\frac{48 \sqrt{2}}{17}, & t \neq 1, s=1 \\ \left(\frac{8 \sqrt{5}}{9}+\frac{8 \sqrt{10}}{13}+\frac{48 \sqrt{2}}{17}+5\right) t+\frac{51-48 \sqrt{2}}{17}, & \\ 9 s t+\left(\frac{16 \sqrt{10}}{13}+\frac{48 \sqrt{2}}{17}-4\right) s+\left(\frac{8 \sqrt{5}}{9}\right. & \\ \left.+\frac{8 \sqrt{10}}{13}+\frac{48 \sqrt{2}}{17}-4\right) t+3+\frac{16 \sqrt{5}}{9} & t \neq 1, s>1 \\ -\frac{16 \sqrt{10}}{13}-\frac{96 \sqrt{2}}{17}, & \end{cases}
$$

Proof. Let $e_{i, j}$ denotes the number of edges of the line graph of the subdivision graph of graphene with $i=s_{u}$ and $j=s_{v}$. It is easy to see that the summation of degree of edge endpoints of graphene has seven edge types $e_{4,4}, e_{4,5}, e_{5,5}, e_{5,8}, e_{8,8}, e_{8,9}$ and $e_{9,9}$ that are shown in Table 8.

Case 1. The fifth $G A$ index of the line graph of the subdivision graph of Graphene for $t=1$ and $s>1$,

$$
\begin{aligned}
G A_{5}(G)= & \sum_{u v \in E(G)} \frac{2 \sqrt{s_{u} s_{v}}}{s_{u}+s_{v}} \\
= & e_{4,4}\left(\frac{2 \sqrt{4 \times 4}}{4+4}\right)+e_{4,5}\left(\frac{2 \sqrt{4 \times 5}}{4+5}\right)+e_{5,5}\left(\frac{2 \sqrt{5 \times 5}}{5+5}\right)+e_{5,8}\left(\frac{2 \sqrt{5 \times 8}}{5+8}\right) \\
& +e_{8,8}\left(\frac{2 \sqrt{8 \times 8}}{8+8}\right)+e_{8,9}\left(\frac{2 \sqrt{8 \times 9}}{8+9}\right)+e_{9,9}\left(\frac{2 \sqrt{9 \times 9}}{9+9}\right)
\end{aligned}
$$


By using Table 8, we get

$$
\begin{aligned}
G A_{5}(G)= & 10\left(\frac{2 \times 2 \times 2}{8}\right)+4\left(\frac{2 \times 2 \sqrt{5}}{9}\right)+2(s-2)\left(\frac{2 \times 5}{10}\right) \\
& +4(s-1)\left(\frac{2 \times 2 \sqrt{10}}{13}\right)+2(s-1)\left(\frac{2 \times 8}{16}\right)+4(s-1)\left(\frac{2 \times 2 \times 3 \sqrt{2}}{17}\right) \\
& +(s-1)\left(\frac{2 \times 9}{18}\right) \\
= & \left(5+\frac{16 \sqrt{10}}{13}+\frac{48 \sqrt{2}}{17}\right) s+3+\frac{16 \sqrt{5}}{9}-\frac{16 \sqrt{10}}{13}-\frac{48 \sqrt{2}}{17} .
\end{aligned}
$$

Case 2. For $t \neq 1$ and $s=1$, then the geometric-arithmetic index of the line graph of the subdivision graph of graphene is

$$
\begin{aligned}
G A_{5}(G)= & \sum_{u v \in E(G)} \frac{2 \sqrt{s_{u} s_{v}}}{s_{u}+s_{v}} \\
= & e_{4,4}\left(\frac{2 \sqrt{4 \times 4}}{4+4}\right)+e_{4,5}\left(\frac{2 \sqrt{4 \times 5}}{4+5}\right)+e_{5,8}\left(\frac{2 \sqrt{5 \times 8}}{5+8}\right)+e_{8,8}\left(\frac{2 \sqrt{8 \times 8}}{8+8}\right) \\
& +e_{8,9}\left(\frac{2 \sqrt{8 \times 9}}{8+9}\right)+e_{9,9}\left(\frac{2 \sqrt{9 \times 9}}{9+9}\right)
\end{aligned}
$$

By using Table 7

$$
\begin{aligned}
G A_{5}(G)= & (t+8)\left(\frac{2 \times 2 \times 2}{8}\right)+2 t\left(\frac{2 \times 2 \sqrt{5}}{9}\right)+2 t\left(\frac{2 \times 2 \sqrt{10}}{13}\right)+2\left(\frac{2 \times 8}{16}\right) \\
& +4(t-1)\left(\frac{2 \times 2 \times 3 \sqrt{2}}{17}\right)+(4 t-7)\left(\frac{2 \times 9}{18}\right) \\
= & \left(\frac{8 \sqrt{5}}{9}+\frac{8 \sqrt{10}}{13}+\frac{48 \sqrt{2}}{17}+5\right) t+\frac{51-48 \sqrt{2}}{17} .
\end{aligned}
$$

Case 3. For $t \neq 1$ and $s>1$, then the geometric-arithmetic index of the line graph of the subdivision of graphene is

$$
\begin{aligned}
G A_{5}(G)= & \sum_{u v \in E(G)} \frac{2 \sqrt{s_{u} s_{v}}}{s_{u}+s_{v}} \\
= & e_{4,4}\left(\frac{2 \sqrt{4 \times 4}}{4+4}\right)+e_{4,5}\left(\frac{2 \sqrt{4 \times 5}}{4+5}\right)+e_{5,5}\left(\frac{2 \sqrt{5 \times 5}}{5+5}\right)+e_{5,8}\left(\frac{2 \sqrt{5 \times 8}}{5+8}\right) \\
& +e_{8,8}\left(\frac{2 \sqrt{8 \times 8}}{8+8}\right)+e_{8,9}\left(\frac{2 \sqrt{8 \times 9}}{8+9}\right)+e_{9,9}\left(\frac{2 \sqrt{9 \times 9}}{9+9}\right)
\end{aligned}
$$


By using Table 6

$$
\begin{aligned}
G A_{5}(G)= & (t+6)\left(\frac{2 \times 2 \times 2}{8}\right)+2(t+2)\left(\frac{2 \times 2 \sqrt{5}}{9}\right)+2(s-2)\left(\frac{2 \times 5}{10}\right) \\
& +2(2 s+t-2)\left(\frac{2 \times 2 \sqrt{10}}{13}\right)+2 s\left(\frac{2 \times 8}{16}\right) \\
& +4(s+t-2)\left(\frac{2 \times 2 \times 3 \sqrt{2}}{17}\right)+(9 s t-8 s-5 t+1)\left(\frac{2 \times 9}{18}\right) \\
G A_{5}(G)= & 9 s t+\left(\frac{16 \sqrt{10}}{13}+\frac{48 \sqrt{2}}{17}-4\right) s+\left(\frac{8 \sqrt{5}}{9}+\frac{8 \sqrt{10}}{13}+\frac{48 \sqrt{2}}{17}-4\right) t+3 \\
& +\frac{16 \sqrt{5}}{9}-\frac{16 \sqrt{10}}{13}-\frac{96 \sqrt{2}}{17} .
\end{aligned}
$$

Case 4. For $t=1$ and $s=1$, then the geometric-arithmetic index of the line graph of the subdivision of graphene is

$$
\begin{aligned}
G A_{5}(G) & =\sum_{u v \in E(G)} \frac{2 \sqrt{s_{u} s_{v}}}{s_{u}+s_{v}} \\
& =12\left(\frac{2 \sqrt{4 \times 4}}{4+4}\right) \\
& =12 .
\end{aligned}
$$

\section{Conclusion}

In this paper, we have computed certain degree based indices, namely first general Zagreb index $M_{\alpha}$, general Randić connectivity index $R_{\alpha}$, general sum-connectivity index $\chi_{\alpha}$, atom-bond connectivity index, geometric-arithmetic index, fourth version of atombond connectivity index $A B C_{4}$ and fifth version of geometric-arithmetic index $G A_{5}$ of the line graph of subdivision of honeycomb networks and graphene. These topological indices correlate certain physico-chemical properties like boiling point, enthalpy of vaporization, strain energy, stability, etc., of chemical compounds. This will start a new direction in this field. However, there are still remains open and challenging problem for researchers.

\section{Acknowledgements}

The authors would like to thank the referees for their constructive suggestions and useful comments which resulted in an improved version of this paper. This research is supported by the start up research grant 2016 of United Arab Emirates University, Al Ain, United Arab Emirates via Grant No. G00002233.

\section{References}

[1] S.; Akhter, M.; Imran, "On degree based topological descriptors of strong product graphs", Can. J. Chem., 94(6), 559-565(2016).

[2] A.; Ali, Z.; Raza, A. A.; Bhatti, "Extremal pentagonal chains with respect to bond incident degree indices", Can. J. Chem., 94(10), 870-876(2016).

[3] A.; Ali, A. A.; Bhatti, Z.; Raza, "Topological study of tree-like polyphenylene systems, spiro hexagonal systems and polyphenylene dendrimer nanostars", Quantum Matter, 5(4), 534-538(2016).

[4] A.; Ali, Z.; Raza, A. A.; Bhatti, "Bond incident degree (BID) indices, nanostructures", Optoelectronics Adv. Materials-Rapid Commun, 10, 1-2(2016). 
[5] A.; Ali, Z.; Raza, A. A.; Bhatti, "Bond Incident Degree (BID) Indices of Polyomino Chains: A Unified Approach", Appl. Math. Comput., 287(9), 28-37(2016).

[6] A.; Ali, Z.; Raza, A. A.; Bhatti, "On the Augmented Zagreb Index", Kuwait Journal of Science, 43(2), 123-138(2016).

[7] A.; Ali, A. A.; Bhatti, Z.; Raza, "The augmented Zagreb index, vertex connectivity and matching number of graphs", Bull. Iranian Math. Soc. 42(2), 417-425(2016).

[8] M.; Bača, J.; Horvàthovà, M.; Mokrišovà and A.; Suhànyiovà, "On topological indices of fullerenes", Appl. Math. Comput., 251, 154-161(2015).

[9] E.; Estrada, L.; Torres, L.; Rodriguez and I.; Gutman, "An atom-bond connectivity index: modelling the enthalpy of formation of alkanes", Ind. J. Chem., 37A, 849-855(1998).

[10] M.; Ghorbani and M. A.; Hosseinzadeh, "Computing $A B C_{4}$ index of nanostar dendrimers", Optoelectron. Adv. Mater.-Rapid Commun., 4(9), 1419-1422(2010).

[11] A.; Graovac, M.; Ghorbani and M. A.; Hosseinzadeh, "Computing fifth geometricarithmetic index for nanostar dendrimers", J. Math. Nanosci., 1(1), 33-42(2011).

[12] S.; Hayat and M.; Imran, "Computation of certain topological indices of nanotubes covered by $C_{5}$ and $C_{7}$ ", J. Comput. Theor. Nanosci., 12(4), 533-541(2015).

[13] S.; Hayat and M.; Imran, "On some degree based topological indices of certain nanotubes", J. Comput. Theor. Nanosci., 12(8), 1599-1605(2015).

[14] S.; Hayat and M.; Imran, "Computation of certain topological indices of nanotubes", J. Comput. Theor. Nanosci., 12(1), 70-76(2015).

[15] M.; Imran and S.; Hayat, "Computation of topological indices of certain networks", Appl. Math. Comput., 240, 213-228(2014).

[16] M.; Imran, S.; Hayat and M. Y. H.; Malik, "On topological indices of certain interconnection networks", Appl. Math. Comput., 244, 936-951(2014).

[17] X.; Li and H.; Zhao, "Trees with the first three smallest and largest generalized topological indices", MATCH Commun. Math. Comput. Chem., 50, 57-62(2004).

[18] M. F.; Nadeem, S.; Zafar and Z.; Zahid, "On certain topological indices of the line graph of subdivision graphs", Appl. Math. Comput., 271, 790-794(2015).

[19] M. F.; Nadeem, S.; Zafar and Z.; Zahid, "On topological properties of the line graphs of subdivision graphs of certain nanostructures", Appl. Math. Comput., 273, 125-130(2016).

[20] Z.; Raza, "The edge version of geometric arithmetic index of polyomino chains of 8-cycles and arbitrary carbon nanocones", J. Comput. Theor. Nanosci., 13, 1-5(2016).

[21] Z.; Raza, A. A.; Bhatti, A.; Ali, "More on Comparison Between First Geometric-Arithmetic Index and Atom-Bond Connectivity Index", Miskolc Mathematical Notes, 17(1), 561$570(2016)$.

[22] M.; Randić, "On characterization of molecular branching", J. Am. Chem. Soc., 97, 66096615(1975).

[23] B.; Rajan, A.; William, C.; Grigorious and S.; Stephen, "On certain topological indices of silicate, honeycomb and hexagonal networks", J. Comp. Math. Sci., 5, 530-535(2012).

[24] P. S.; Ranjini, V.; Lokesha and I. N.; Cangl, "On the Zagreb indices of the line graphs of the subdivision graphs", Appl. Math. Comput., 18, 699-702(2011).

[25] P. S.; Ranjini, V.; Lokesha and M. A.; Rajan, "On the Shultz index of the subdivision graphs", Adv. Stud. Contemp. Math., 21(3), 279-290(2011).

[26] G.; Su and L.; Xu, "Topological indices of the line graph of subdivision graphs and their Schur-bounds", Appl. Math. Comput., 253, 395-401(2015).

[27] G.; Sridhara, M. R.; Rajesh Kanna and R. S.; Indumathi, "Computation of Topological Indices of Graphene" J. Nanomaterials, (2015).

[28] D.; Vukičević and B.; Furtula, "Topological index based on the ratios of geometrical and arithmetical mmeans of end-vertex degrees of edges" J. Math. Chem., 46, 1369-1376(2009).

[29] B.; Zhou and N.; Trinajstić, "On a novel connectivity index", J. Math. Chem., 46, 12521270(2009).

[30] B.; Zhou and N.; Trinajstić, "On general sum-connectivity index", J. Math. Chem., 47, 210-218(2010). 
